\title{
Clinical performance of decellularized heart valves versus standard tissue conduits: a systematic review and meta-analysis
}

\author{
Steve W. F. R. Waqanivavalagi ${ }^{1,2^{*}}$ D, Sameer Bhat ${ }^{1,3}$, Marcus B. Ground ${ }^{1,4}$, Paget F. Milsom ${ }^{5}$ and Jillian Cornish ${ }^{1}$
}

\begin{abstract}
Background: Valve replacement surgery is the definitive management strategy for patients with severe valvular disease. However, valvular conduits currently in clinical use are associated with significant limitations. Tissueengineered (decellularized) heart valves are alternative prostheses that have demonstrated promising early results. The purpose of this systematic review and meta-analysis is to perform robust evaluation of the clinical performance of decellularized heart valves implanted in either outflow tract position, in comparison with standard tissue conduits.

Methods: Systematic searches were conducted in the PubMed, Scopus, and Web of Science databases for articles in which outcomes between decellularized heart valves surgically implanted within either outflow tract position of human subjects and standard tissue conduits were compared. Primary endpoints included postoperative mortality and reoperation rates. Meta-analysis was performed using a random-effects model via the Mantel-Haenszel method.

Results: Seventeen articles were identified, of which 16 were included in the meta-analysis. In total, 1418 patients underwent outflow tract reconstructions with decellularized heart valves and 2725 patients received standard tissue conduits. Decellularized heart valves were produced from human pulmonary valves and implanted within the right ventricular outflow tract in all cases. Lower postoperative mortality (4.7\% vs. 6.1\%; RR 0.94, 95\% Cl: 0.60-1.47; $P=$ 0.77 ) and reoperation rates (4.8\% vs. $7.4 \%$; RR $0.55,95 \% \mathrm{Cl}: 0.36-0.84 ; P=0.0057)$ were observed in patients with decellularized heart valves, although only reoperation rates were statistically significant. There was no statistically significant heterogeneity between the analyzed articles $\left(P^{2}=31 \%, P=0.13\right.$ and $P^{2}=33 \%, P=0.10$ respectively).

Conclusions: Decellularized heart valves implanted within the right ventricular outflow tract have demonstrated significantly lower reoperation rates when compared to standard tissue conduits. However, in order to allow for more accurate conclusions about the clinical performance of decellularized heart valves to be made, there need to be more high-quality studies with greater consistency in the reporting of clinical outcomes.
\end{abstract}

Keywords: Decellularization, Tissue engineering, Valve replacement

\footnotetext{
* Correspondence: s.waqanivavalagi@auckland.ac.nz

'Department of Medicine, Faculty of Medical and Health Sciences, University of Auckland, Grafton, Auckland 1023, New Zealand

${ }^{2}$ Adult Emergency Department, Auckland City Hospital, Auckland District Health Board, Grafton, Auckland 1023, New Zealand

Full list of author information is available at the end of the article
}

(c) The Author(s). 2020 Open Access This article is licensed under a Creative Commons Attribution 4.0 International License, which permits use, sharing, adaptation, distribution and reproduction in any medium or format, as long as you give appropriate credit to the original author(s) and the source, provide a link to the Creative Commons licence, and indicate if changes were made. The images or other third party material in this article are included in the article's Creative Commons licence, unless indicated otherwise in a credit line to the material. If material is not included in the article's Creative Commons licence and your intended use is not permitted by statutory regulation or exceeds the permitted use, you will need to obtain permission directly from the copyright holder. To view a copy of this licence, visit http://creativecommons.org/licenses/by/4.0/ The Creative Commons Public Domain Dedication waiver (http://creativecommons.org/publicdomain/zero/1.0/) applies to the data made available in this article, unless otherwise stated in a credit line to the data. 


\section{Introduction}

Valve replacement surgery is the definitive management strategy for patients with advanced valvular disease [1$3]$. However, the conduits used at operation have each faced some limitation precluding their routine clinical use. For example, although mechanical valves are often selected for younger patients because of their longlasting durability [4, 5], their higher thromboembolic risk means that patients require lifelong anticoagulation, which carries a risk of life-threatening hemorrhage [68]. Thus, for women of child-bearing age or the elderly, a bioprosthetic valve might be selected instead, thereby obviating the need for anticoagulants [9]. Despite this advantage, bioprosthetic valves elicit an inflammatory response that leads to progressive calcification, graft failure, and earlier reintervention $[8,10]$. This inflammatory reaction is especially profound in children, which is why homografts are often selected as the conduits of choice for pediatric patients [11]. Nevertheless, homografts remain susceptible to graft-versushost disease, lack regenerative capacity, and are often poorly available [12]. Thus, an ideal valvular prosthesis is yet to be identified [13-16].

The characteristics of an ideal valve replacement prosthesis would be: durability $[14,17,18]$, nonimmunogenicity $[15,19]$, non-thrombogenicity $[16,20]$, capability of growth, self-repair and remodeling [15-19], and lasting the recipient's lifespan [15]. In an attempt to achieve this goal, tissue-engineered heart valves have been designed using various combinations of the following framework: firstly, the genetic material of the xenograft is removed through a decellularization process that produces an acellular connective tissue scaffold upon which host cells are seeded [21, 22]. The valve is then conditioned in a bioreactor before surgical implantation $[23,24]$. It is anticipated that the finished product is a living tissue conduit that meets the requirements of an ideal valve prosthesis.

Unfortunately, early clinical trials of decellularized grafts have produced catastrophic results. A report on the clinical performance of the SynerGraft ${ }^{\text {ts }}$ porcine valve implanted within pediatric patients revealed that the graft elicited a strong inflammatory response with fatal consequences soon after implantation [25]. However, these outcomes are not universal. Konertz et al. reported that the Matrix $\mathrm{P}^{\mathrm{TM}}$ graft, a decellularized porcine xenograft, had a similar physiological performance to the native valve [26]. Furthermore, significant improvements in decellularization technology have been achieved since the complications following graft implantation in humans first attracted concern [27, 28] and decellularized heart valves have since been examined in large animal models and humans with encouraging outcomes [29-31].
Heart valve tissue-engineering has now matured to the point where various products have been brought to clinical trial [27, 32-34]. However, the performance of these conduits is yet to be systematically evaluated. This systematic review and meta-analysis was undertaken to: (1) record, assess and discuss the collective clinical performance of decellularized heart valves compared with standard conduits; (2) establish whether groups that have investigated the clinical utility of decellularized heart valves report similar categories of findings, and, if not; (3) develop a recommendation for outcomes that future groups might consider when publishing the results of their studies.

\section{Methods}

This systematic review was conducted in accordance with the Preferred Reporting Items for Systematic Reviews and Meta-Analyses (PRISMA) guidelines [35]. Two reviewers (S.B. and S.W.) systematically searched medical databases, assessed the titles, abstracts, and full texts of articles, extracted the data, and performed quality assessments using standard quality assessment tools.

\section{Literature search strategy}

PubMed, Scopus and Web of Science electronic databases were searched from their dates of inception to September 2019. The search terms "decellularization", "acellularization" and "valve" were combined as either keywords or Medical Subject Headings (MeSH) terms using the Boolean operators "AND" and "OR" in the following format: [decellular* OR acellular*] AND valv*. There were no date or geographic restrictions on search results. Titles and abstracts of the retrieved results were independently assessed, and reference lists of the extracted publications were manually reviewed to capture all potentially relevant studies. The full text of each possibly relevant article was independently appraised. Eligible articles were then set aside, and any disagreements were resolved by consensus, prior to finalizing the list of included texts for systematic review.

\section{Study selection}

Articles were selected for inclusion if they were written in English and reported any of the clinical or preclinical outcomes for decellularized heart valves surgically implanted within the outflow tract position of human subjects. Studies were excluded if the decellularization protocol for heart valves was not clearly outlined or if a previously published decellularization protocol was not adequately referenced, there was no control or comparison group, or if the outcomes for the decellularized group of subjects was not separately analyzed. There were no restrictions on study design, study size, date, or geographic location. 
Abstracts, book chapters, case reports, conference presentations, retracted articles, editorials, letters to the editor, commentaries, and expert opinions were excluded. Review articles were omitted to avoid publication bias and the duplication of results. Articles were also excluded if the full text could not be sourced.

\section{Data extraction}

Data were recorded in a Microsoft Excel ${ }^{\circ}$ (Microsoft Corporation, Redmond, Washington, US) proforma spreadsheet. The following agreed data points were recorded from each article: article characteristics (authors, year of publication, study design, and geographic location); basic demographic characteristics (total and decellularized valve sample sizes, gender, age, and mass); indication(s) for, and characteristics of, the decellularized heart valve replacements; the heart valve decellularization protocol employed; and the pre-determined primary and secondary endpoints (Table 1).

\section{Quality appraisal}

Appraisals of the methodological quality of included observational cohort studies were performed using the Newcastle-Ottawa Quality Assessment Scale [36]. This scale assigns a score to each of three quality assessment domains using a 'star system'. Awarding a star indicates the presence of a particular study design characteristic (refer to Additional file 1). High-quality studies were those achieving seven or more stars, medium-quality were those achieving four to six stars, and low-quality were those achieving fewer than four stars.

The risk of bias within the included nonrandomized interventional studies was identified using the Risk of Bias In Non-Randomized Studies of Interventions (ROBINS-I) tool [37]. The ROBINS-I tool divides each study into seven bias domains. Each of these bias domains was classified as at either low, moderate, serious, or critical risk of bias. An overall judgment of the risk of bias for each study was then made using an identical assessment rubric. Studies with an overall "low" risk of bias were considered equivalent to a well-performed randomized controlled trial (RCT).

\section{Statistical analysis}

Meta-analysis was performed for the primary endpoints (postoperative mortality and reoperation rates) using the meta package with $\mathrm{R}$ software (Version 4.0.0; R Foundation for Statistical Computing, Vienna, Austria). The Mantel-Haenszel method was used to calculate the pooled relative risk (RR) and 95\% confidence interval (CI) for the binary primary endpoints (mortality versus no mortality and reoperation versus no reoperation). Statistical heterogeneity between studies was evaluated using the Cochran Q test and then quantified using the $I^{2}$ test statistic [38]. We opted for a random-effects rather than a fixed-effects model, due to the expected level of heterogeneity between the studies included in the meta-analysis. Publication biases were assessed using Egger's regression test and by generating funnel plots. Sensitivity analyses were performed by excluding studies at moderate or serious risk of bias, which may have had large heterogeneity, and studies with very large sample sizes, which may have disproportionately influenced the pooled summary estimate. Secondary endpoints and basic demographic data were synthesized qualitatively, using probability $(\mathrm{P})$ values, total numbers and percentages where appropriate. $P$-values $<0.05$ were considered statistically significant for all reported outcomes.

Table 1 Pre-determined primary and secondary endpoints extracted from eligible full-text articles

\begin{tabular}{|c|c|c|c|c|c|c|}
\hline \multicolumn{2}{|c|}{ Primary endpoints } & \multicolumn{5}{|c|}{ Secondary endpoints } \\
\hline Mortality & Reoperations & $\begin{array}{l}\text { Transvalvular } \\
\text { gradient }\end{array}$ & Valvular function & Valvular characteristics & $\begin{array}{l}\text { Histological } \\
\text { commentary }\end{array}$ & Adverse events \\
\hline 30-day & 30-day & Overall & Insufficiency & Diameter & Fibroproliferation & $\begin{array}{l}\text { Infective endocarditis } \\
\text { OR freedom from } \\
\text { infective endocarditis }\end{array}$ \\
\hline 1-year & 1-year & & Stenosis & Area & Recellularization & $\begin{array}{l}\text { Valve thrombosis OR } \\
\text { freedom from valve } \\
\text { thrombosis }\end{array}$ \\
\hline \multirow[t]{3}{*}{$\begin{array}{l}\text { Overall rate OR } \\
\text { freedom from } \\
\text { mortality }\end{array}$} & $\begin{array}{l}\text { Overall rate OR } \\
\text { freedom from } \\
\text { reoperations }\end{array}$ & & $\begin{array}{l}\text { Explantation rate } \\
\text { OR freedom from } \\
\text { explantation }\end{array}$ & Calcification & $\begin{array}{l}\text { Antibody/humoral } \\
\text { immune response }\end{array}$ & \\
\hline & Time to reoperation & & $\begin{array}{l}\text { Rate of dysfunction } \\
\text { OR freedom from } \\
\text { dysfunction }\end{array}$ & Cusp mobility & & \\
\hline & & & $\begin{array}{l}\text { Failure rate } O R \\
\text { freedom from failure }\end{array}$ & Cusp retraction & & \\
\hline
\end{tabular}




\section{Results}

\section{Quantity of evidence}

The search of medical databases revealed 2306 abstracts, from which 102 full-text articles were assessed for their eligibility and inclusion (PRISMA Diagram: Fig. 1). In total, 17 full-text articles were included for qualitative analysis. All but one study were included for metaanalysis of primary endpoints [39]. The characteristics of each of these articles are provided in Table 2.

The articles selected for data analysis were conducted across four countries, most frequently within the United States of America, and they spanned a 16-year interval, from 2003 to 2019. The published experiences of outflow tract reconstructions using decellularized heart valves were equally divided between interventional and observational cohort studies $(n=9$ and $n=8$ studies, respectively). The observational cohort studies were largely retrospective ( $n=7$ studies), with a single study conducted prospectively (Table 2). There were no RCT.

\section{Quality of evidence}

All included observational cohort studies were judged as high-quality, based on their Newcastle-Ottawa Quality Assessment scores (refer to Additional file 2).

Only two of the nine $(\sim 22 \%)$ included non-randomized interventional studies were judged as being at "low" risk of bias, and hence, were comparable to a well-performed RCT $[48,49]$. The remaining studies were judged as at either "moderate" or "serious" risk of bias $(n=4$ and $n=3$ studies, respectively), with such judgment being made principally on the basis of either bias due to confounding or missing data $[33,39,40]$ (refer to Additional file 3 ).

\section{Basic demographics \\ Population descriptors}

Outflow tract reconstructions were performed in 4143 patients, of whom 1418 (34.2\%) patients received decellularized heart valves and 2725 (65.8\%) patients received

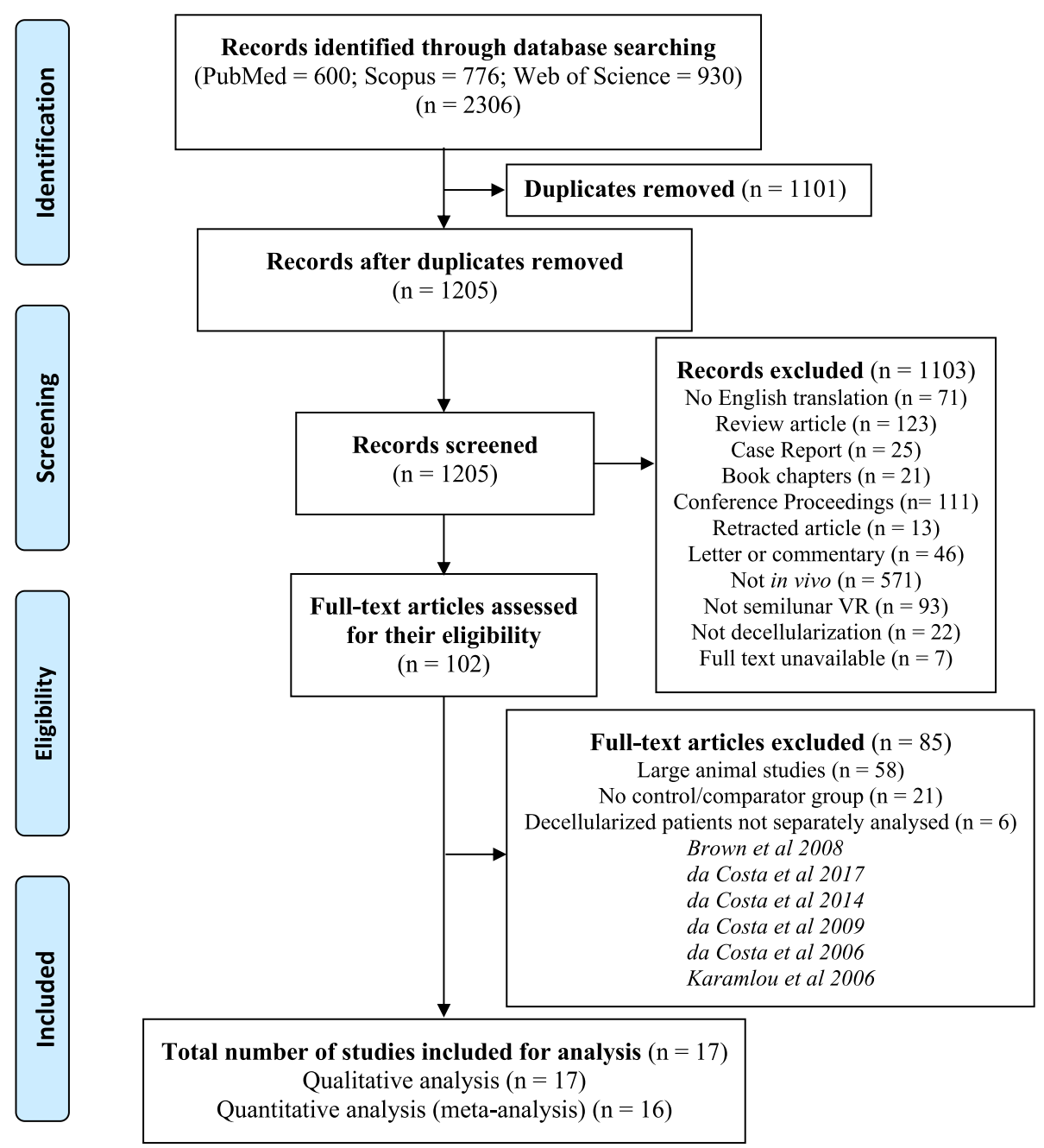

Fig. 1 PRISMA flow diagram demonstrating the full-text article selection process 
Table 2 Characteristics of eligible full-text articles

\begin{tabular}{lllll}
\hline Authors & Year & Study design & Retrospective/prospective & Geographic location \\
\hline Bechtel et al. [39] & 2005 & Interventional, non-randomized controlled trial & Prospective & Germany \\
Bechtel et al. [40] & 2008 & Interventional, non-randomized controlled trial & Prospective & Germany \\
Bibevski et al. [41] & 2017 & Observational cohort study & Retrospective & United States of America \\
Boethig et al. [42] & 2019 & Observational cohort study & Prospective & Germany \\
Brown et al. [43] & 2010 & Observational cohort study & Retrospective & United States of America \\
Brown et al. [44] & 2011 & Observational cohort study & Retrospective & United States of America \\
Burch et al. [45] & 2010 & Observational cohort study & Retrospective & United States of America \\
Cebotari et al. [46] & 2011 & Interventional, non-randomized controlled trial & Prospective & Germany \\
da Costa et al. [47] & 2005 & Interventional, non-randomized controlled trial & Prospective & Brazil \\
da Costa et al. [33] & 2007 & Interventional, non-randomized controlled trial & Prospective & Brazil \\
da Costa et al. [48] & 2018 & Interventional, non-randomized controlled trial & Prospective & Brazil \\
Etnel et al. [49] & 2018 & Interventional, non-randomized controlled trial & Prospective & Netherlands \\
Konuma et al. [50] & 2009 & Observational cohort study & Retrospective & United States of America \\
Ruzmetov et al. [51] & 2012 & Observational cohort study & Retrospective & United States of America \\
Sarikouch et al. [34] & 2016 & Interventional, non-randomized controlled trial & Prospective & Germany \\
Sievers et al. [52] & 2003 & Interventional, non-randomized controlled trial & Prospective & Retrospective \\
Tavakkol et al. [53] & 2005 & Observational cohort study & Germany
\end{tabular}

standard tissue conduits (refer to population specific data provided in Table 3 ).

\section{Semilunar heart valve replacement descriptors}

The indications for outflow tract reconstruction surgery were reported in 11 of the 17 included articles. In descending order of frequency, these indications were: congenital heart disease, mixed (stenotic and regurgitant) valvular disease, valvular regurgitation, valvular stenosis, and infective endocarditis (Additional file 4).

The decellularized heart valves comprised pulmonary valve tissue obtained from human donors and implantation of the grafts was restricted to human recipients in all studies. Decellularized pulmonary valves were implanted within the right ventricular outflow tract in each article. Thus, no cases of left ventricular outflow tract reconstruction were reported (Table 4).

\section{Decellularization protocols for semilunar heart valves}

The decellularization protocol for pulmonary valve homografts was adequately described or referenced in all articles. Three articles described novel decellularization processes that typically comprised: $0.9 \%$ sodium chloride, the detergents sodium deoxycholate and sodium dodecylsulfate, penicillin and streptomycin as the sole combination of antimicrobial agents, and incubation at temperatures ranging from $-150{ }^{\circ} \mathrm{C}$ to $20^{\circ} \mathrm{C}[33,34,46]$. In the remainder of articles, valves were decellularized using one of three previously patented or published protocols [27, 28, 54] (Table 5). The protocol developed by
CryoLife Inc. (CryoLife Inc., Kennesaw, Georgia, US) was employed in 10 of the remaining 14 articles [54].

\section{Primary endpoints (Additional file 5) Postoperative mortality}

Fifteen of the included studies reported postoperative mortality rates. Two of the studies demonstrated statistically significant results, with lower postoperative mortality rates in patients with decellularized heart valves [43, 49]. Two included studies did not report any instances of mortality and thus did not contribute to the pooled summary estimate [44, 47]. Overall, lower postoperative mortality rates were observed in patients with decellularized heart valves, when compared to patients with standard tissue conduits, although these findings were not statistically significant (4.7\% vs. $6.1 \%$; pooled RR 0.94 , 95\% CI: 0.60 to $1.47 ; P=0.77$; Fig. 2 ). There was no significant heterogeneity between the analyzed studies $\left(I^{2}=31 \% ; P=0.13\right)$.

\section{Reoperation}

Fifteen included studies reported reoperation rates. Three of the studies demonstrated statistically significant results, with lower reoperation rates observed for patients with decellularized heart valves [41, 48, 49]. Overall, significantly lower reoperation rates were observed in patients with decellularized heart valves, when compared to patients with standard tissue conduits $(4.8 \%$ vs. $7.4 \%$; pooled RR 0.55 , 95\% CI: 0.36 to $0.84 ; P=0.0057$; Fig. 3 ). There was no significant heterogeneity between the analyzed studies $\left(I^{2}=33 \% ; P=0.10\right)$. 
Table 3 Population descriptors of eligible full-text articles

\begin{tabular}{|c|c|c|c|c|c|c|}
\hline Authors & $\begin{array}{l}\text { Total sample } \\
\text { size }\end{array}$ & $\begin{array}{l}\text { Standard tissue } \\
\text { conduit sample } \\
\text { size }\end{array}$ & $\begin{array}{l}\text { Decellularized } \\
\text { valve sample } \\
\text { size }\end{array}$ & $\operatorname{Sex}(M ; F)^{a}$ & $\begin{array}{l}\text { [Median age]; } \\
\text { mean age } \pm \text { SD } \\
(\text { range), years }\end{array}$ & $\begin{array}{l}\text { [Median mass]; } \\
\text { mean mass } \pm \text { SD } \\
\left(\text { range), } \mathrm{kg}^{\mathrm{a}}\right.\end{array}$ \\
\hline Bechtel et al. 2005 [39] & 69 & 47 & 22 & $18 ; 4$ & $37.4 \pm 10.2$ & \\
\hline Bechtel et al. 2008 [40] & 72 & 49 & 23 & $19 ; 4$ & $37.0 \pm 10.8$ & \\
\hline Bibevski et al. 2017 [41] & 287 & 124 & 163 & $107 ; 56$ & $17.3 \pm 16.4(0.005-74)$ & $45.8 \pm 36.3(2-126)$ \\
\hline Boethig et al. 2019 [42] & 705 & 470 & 235 & $136 ; 99$ & $19.1 \pm 12.8$ & \\
\hline Brown et al. 2010 [43] & 1588 & 1246 & 342 & $229 ; 113$ & $23.9 \pm 12.6(0-69.6)$ & \\
\hline Brown et al. 2011 [44] & 63 & 34 & 29 & & {$[22] ; 28.6 \pm 16.0(0.25-58)$} & {$[81] ; 79.3 \pm 28.4(5-126)$} \\
\hline Burch et al. 2010 [45] & 94 & 47 & 47 & & {$[9.11] ; 9.91 \pm 8.08(0.011-30.03)$} & {$[25] ; 33 \pm 26(3-90)$} \\
\hline Cebotari et al. 2011 [46] & 114 & 76 & 38 & $16 ; 22$ & $16.4 \pm 11.4$ & \\
\hline da Costa et al. 2005 [47] & 20 & 9 & 11 & $5 ; 6$ & $23.0 \pm 9.04(9-37)$ & \\
\hline da Costa et al. 2007 [33] & 136 & 68 & 68 & $48 ; 20$ & $30.3 \pm 11.2(9-56)$ & \\
\hline da Costa et al. 2018 [48] & 188 & 94 & 94 & $74 ; 20$ & {$[34.0]$} & \\
\hline Etnel et al. 2018 [49] & 260 & 130 & 130 & $93 ; 37$ & {$[28]$} & \\
\hline Konuma et al. 2009 [50] & 82 & 41 & 41 & & $5.5 \pm 7.4$ & $20.5 \pm 22.0$ \\
\hline Ruzmetov et al. 2012 [51] & 100 & 61 & 39 & $25 ; 14$ & $19.2 \pm 17.2(0.02-72)$ & $54.7 \pm 40.4(2.8-167)$ \\
\hline Sarikouch et al. 2016 [34] & 279 & 186 & 93 & $58 ; 35$ & $15.8 \pm 10.2$ & \\
\hline Sievers et al. 2003 [52] & 34 & 17 & 17 & $13 ; 4$ & $39.7 \pm 9.7$ & \\
\hline Tavakkol et al. 2005 [53] & 52 & 26 & 26 & & $5.1 \pm 5.4$ & $20.5 \pm 22.7$ \\
\hline
\end{tabular}

Blank cells correspond with data points that were not reported in the respective article

$F$ female, kg kilogram, M male, SD standard deviation

a These data relate to the decellularized heart valve patient population

Table 4 Decellularized heart valve characteristics in eligible full-text articles

\begin{tabular}{|c|c|c|c|c|c|}
\hline Authors & Donor species & Donor tissue/valve type & Recipient species & Recipient site & Number of patients \\
\hline Bechtel et al. 2005 [39] & Human & PV & Human & RVOT & 22 \\
\hline Bechtel et al. 2008 ${ }^{\mathrm{a}}$ [40] & Human & PV & Human & RVOT & 23 \\
\hline Bibevski et al. 2017 [41] & Human & PV & Human & RVOT & 163 \\
\hline Boethig et al. 2019 [42] & Human & PV & Human & RVOT & 235 \\
\hline Brown et al. $2010^{\mathrm{a}}$ [43] & Human & PV & Human & RVOT & 342 \\
\hline Brown et al. $2011^{\mathrm{a}}$ [44] & Human & PV & Human & RVOT & 29 \\
\hline Burch et al. 2010a [45] & Human & PV & Human & RVOT & 47 \\
\hline Cebotari et al. 2011 [46] & Human & PV & Human & RVOT & 38 \\
\hline da Costa et al. 2005 [47] & Human & PV & Human & RVOT & 11 \\
\hline da Costa et al. 2007b [33] & Human & PV & Human & RVOT & 68 \\
\hline da Costa et al. 2018 [48] & Human & PV & Human & RVOT & 94 \\
\hline Etnel et al. $2018^{c}$ [49] & Human & PV & Human & RVOT & 130 \\
\hline Konuma et al. 2009 & Human & PV & Human & RVOT & 41 \\
\hline Ruzmetov et al. 2012 [51] & Human & PV & Human & RVOT & 39 \\
\hline Sarikouch et al. 2016 [34] & Human & PV & Human & RVOT & 93 \\
\hline Sievers et al. 2003 [52] & Human & PV & Human & RVOT & 17 \\
\hline Tavakkol et al. 2005 [53] & Human & PV & Human & RVOT & 26 \\
\hline
\end{tabular}

PV pulmonary valve, RVOT right ventricular outflow tract

${ }^{a}$ CryoValve SynerGraft (CryoLife Inc., Kennesaw, Georgia, US) pulmonary homograft

bPulmonary homografts were provided by the Human Heart Valve Bank (Hospital Santa Casa de Misericórdia, Curitiba, BR)

'Pulmonary homografts were obtained at the Multi-Tissue Bank (Pontifical Catholic University of Paraná, Curitiba Campus, Curitiba, BR) 
Table 5 Decellularization protocol for replacement heart valves in eligible full-text articles

\begin{tabular}{|c|c|c|c|c|}
\hline \multirow[t]{2}{*}{ Authors } & \multicolumn{4}{|c|}{ Decellularization components and conditions } \\
\hline & Detergent(s) & Enzyme(s) & Antimicrobial agent(s) & Temperature \\
\hline Bechtel et al. 2005 [39] & $\begin{array}{l}\text { Hypotonic sterile water } \\
\text { solution (treatment); multi- } \\
\text { day washout period with } \\
\text { isotonic neutral buffer } \\
\text { solution (unspecified) }\end{array}$ & $\begin{array}{l}\text { Ribonuclease }+ \\
\text { deoxyribonuclease }\end{array}$ & $\begin{array}{l}\text { Antibiotic + antimycotic } \\
\text { (unspecified) }\end{array}$ & \\
\hline Bechtel et al. 2008 $[40]$ & $\begin{array}{l}\text { Hypotonic sterile water } \\
\text { solution (treatment); multi- } \\
\text { day washout period with } \\
\text { isotonic neutral buffer } \\
\text { solution (unspecified) }\end{array}$ & $\begin{array}{l}\text { Ribonuclease }+ \\
\text { deoxyribonuclease }\end{array}$ & $\begin{array}{l}\text { Antibiotic + antimycotic } \\
\text { (unspecified) }\end{array}$ & \\
\hline Bibevski et al. $2017^{\mathrm{a}}[41]$ & $\begin{array}{l}\text { Hypotonic sterile water } \\
\text { solution (treatment); multi- } \\
\text { day washout period with } \\
\text { isotonic neutral buffer } \\
\text { solution (unspecified) }\end{array}$ & $\begin{array}{l}\text { Ribonuclease }+ \\
\text { deoxyribonuclease }\end{array}$ & $\begin{array}{l}\text { Antibiotic + antimycotic } \\
\text { (unspecified) }\end{array}$ & \\
\hline Boethig et al., 2019 $[42]$ & $\begin{array}{l}0.5 \% \text { sodium deoxycholate } \\
\text { (Sigma Chemical Company, } \\
\text { St Louis, Missouri, USA)+ } \\
0.5 \% \text { sodium dodecylsulfate } \\
\text { (Carl Roth GmbH, Karlsruhe, } \\
\text { Germany); } 0.9 \% \mathrm{NaCl} \\
\text { (washing) }\end{array}$ & & $\begin{array}{l}\text { Penicillin-streptomycin } \\
(100 \mathrm{IU} / \mathrm{mL})^{\mathrm{e}}\end{array}$ & $\begin{array}{l}20^{\circ} \mathrm{C} \text { (treatment); } \\
4^{\circ} \mathrm{C} \text { (storage) }\end{array}$ \\
\hline Brown et al. $2010^{\mathrm{a}}[43]$ & $\begin{array}{l}\text { Hypotonic sterile water } \\
\text { solution (treatment); multi- } \\
\text { day washout period with } \\
\text { isotonic neutral buffer } \\
\text { solution (unspecified) }\end{array}$ & $\begin{array}{l}\text { Ribonuclease }+ \\
\text { deoxyribonuclease }\end{array}$ & $\begin{array}{l}\text { Antibiotic + antimycotic } \\
\text { (unspecified) }\end{array}$ & \\
\hline Brown et al. $2011^{\mathrm{a}}[44]$ & $\begin{array}{l}\text { Hypotonic sterile water } \\
\text { solution (treatment); multi- } \\
\text { day washout period with } \\
\text { isotonic neutral buffer } \\
\text { solution (unspecified) }\end{array}$ & $\begin{array}{l}\text { Ribonuclease }+ \\
\text { deoxyribonuclease }\end{array}$ & $\begin{array}{l}\text { Antibiotic + antimycotic } \\
\text { (unspecified) }\end{array}$ & \\
\hline Burch et al. $2010^{\mathrm{a}}[45]$ & $\begin{array}{l}\text { Hypotonic sterile water } \\
\text { solution (treatment); multi- } \\
\text { day washout period with } \\
\text { isotonic neutral buffer } \\
\text { solution (unspecified) }\end{array}$ & $\begin{array}{l}\text { Ribonuclease }+ \\
\text { deoxyribonuclease }\end{array}$ & $\begin{array}{l}\text { Antibiotic + antimycotic } \\
\text { (unspecified) }\end{array}$ & \\
\hline Cebotari et al. 2011 [46] & $\begin{array}{l}0.5 \% \text { sodium } \\
\text { deoxycholate }+0.5 \% \\
\text { sodium dodecylsulfate } \\
\text { (treatment); } 0.9 \% \mathrm{NaCl} \\
\text { (washing) }\end{array}$ & & $\begin{array}{l}\text { Penicillin-streptomycin } \\
(100 \mathrm{IU} / \mathrm{mL})^{e}\end{array}$ & $\begin{array}{l}20^{\circ} \mathrm{C} \text { (treatment); } \\
4^{\circ} \mathrm{C} \text { (storage) }\end{array}$ \\
\hline da Costa et al. $2005^{c}[47]$ & $\begin{array}{l}1 \text { to } 2 \% \text { sodium } \\
\text { deoxycholic acid } \\
\text { (treatment); PBS in } \\
\text { decreasing concentrations } \\
\text { (rinsing); } 70 \% \text { ethanol; } \\
\text { pulsating PBS (repeating } \\
\text { rinsing) }\end{array}$ & & & $\begin{array}{l}37^{\circ} \mathrm{C} \text { (storage for } 24 \mathrm{~h} \text { ); } \\
20^{\circ} \mathrm{C} \text { (treatment) }\end{array}$ \\
\hline da Costa et al. 2007 [33] & $\begin{array}{l}\text { 10\% dimethyl sulphoxide + } \\
10 \% \text { fetal bovine serum; } \\
0.9 \% \mathrm{NaCl} \text { (thawing) }+10 \% \\
\text { fetal bovine serum (gradual } \\
\text { dilution); continuous shaking } \\
\text { over } 24 \mathrm{~h} \text { with either } 1 \% \\
\text { sodium deoxycholic acid + } \\
80 \% \text { ethanol OR } 0.1 \% \\
\text { sodium dodecylsulfate; } \\
\text { nutrient solution (harvesting) }\end{array}$ & & $\begin{array}{l}\text { Cefoxitin }(240 \mu \mathrm{g} / \mathrm{mL})+ \\
\text { lincomycin }(120 \mu \mathrm{g} / \mathrm{mL})+ \\
\text { vancomycin }(50 \mu \mathrm{g} / \mathrm{mL})+ \\
\text { polymyxin } B(100 \mu \mathrm{g} / \mathrm{mL})\end{array}$ & $\begin{array}{l}4{ }^{\circ} \mathrm{C} \text { (treatment); } \\
\text { Storage within }-150^{\circ} \mathrm{C} \\
\text { liquid nitrogen vapour; } \\
42 \text { to } 50^{\circ} \mathrm{C} \text { (thawing) }\end{array}$ \\
\hline da Costa et al. 2018 ${ }^{d}[48]$ & $\begin{array}{l}0.1 \% \text { sodium dodecylsulfate } \\
\text { (treatment); PBS (storage) }\end{array}$ & & & $\begin{array}{l}37^{\circ} \mathrm{C} \text { (shaking); } 4^{\circ} \mathrm{C} \\
\text { (storage up to } 90 \text { days) }\end{array}$ \\
\hline Etnel et al. 2018 $[49]$ & $\begin{array}{l}0.1 \% \text { sodium dodecylsulfate } \\
\text { (treatment); PBS (storage) }\end{array}$ & & & $\begin{array}{l}37^{\circ} \mathrm{C} \text { (shaking); } 4^{\circ} \mathrm{C} \\
\text { (storage up to } 90 \text { days) }\end{array}$ \\
\hline
\end{tabular}


Table 5 Decellularization protocol for replacement heart valves in eligible full-text articles (Continued)

\begin{tabular}{|c|c|c|c|c|}
\hline \multirow[t]{2}{*}{ Authors } & \multicolumn{4}{|c|}{ Decellularization components and conditions } \\
\hline & Detergent(s) & Enzyme(s) & Antimicrobial agent(s) & Temperature \\
\hline & $\begin{array}{l}\text { solution (treatment); multi- } \\
\text { day washout period with } \\
\text { isotonic neutral buffer } \\
\text { solution (unspecified) }\end{array}$ & deoxyribonuclease & (unspecified) & \\
\hline $\begin{array}{l}\text { Ruzmetov et al. } 2012^{\mathrm{a}} \\
\text { [51] }\end{array}$ & $\begin{array}{l}\text { Hypotonic sterile water } \\
\text { solution (treatment); multi- } \\
\text { day washout period with } \\
\text { isotonic neutral buffer } \\
\text { solution (unspecified) }\end{array}$ & $\begin{array}{l}\text { Ribonuclease }+ \\
\text { deoxyribonuclease }\end{array}$ & $\begin{array}{l}\text { Antibiotic + antimycotic } \\
\text { (unspecified) }\end{array}$ & \\
\hline Sarikouch et al. 2016 [34] & $\begin{array}{l}0.5 \% \text { sodium deoxycholate } \\
\text { (Sigma Chemical Company, } \\
\text { St Louis, Missouri, USA) + 0.5\% } \\
\text { sodium dodecylsulfate (Carl } \\
\text { Roth GmbH, Karlsruhe, } \\
\text { Germany) (treatment); PBS } \\
\text { ( } 6 \text { cycles of washing, } 12 \text { h each) }\end{array}$ & & $\begin{array}{l}\text { Penicillin-streptomycin } \\
(100 \mathrm{IU} / \mathrm{mL})^{e}\end{array}$ & $4^{\circ} \mathrm{C}$ (storage) \\
\hline Sievers et al. $2003^{\mathrm{a}}[52]$ & $\begin{array}{l}\text { Hypotonic sterile water } \\
\text { solution (treatment); multi- } \\
\text { day washout period with } \\
\text { isotonic neutral buffer } \\
\text { solution (unspecified) }\end{array}$ & $\begin{array}{l}\text { Ribonuclease }+ \\
\text { deoxyribonuclease }\end{array}$ & $\begin{array}{l}\text { Antibiotic + antimycotic } \\
\text { (unspecified) }\end{array}$ & \\
\hline Tavakkol et al. $2005^{\mathrm{a}}[53]$ & $\begin{array}{l}\text { Hypotonic sterile water } \\
\text { solution (treatment); multi- } \\
\text { day washout period with } \\
\text { isotonic neutral buffer } \\
\text { solution (unspecified) }\end{array}$ & $\begin{array}{l}\text { Ribonuclease }+ \\
\text { deoxyribonuclease }\end{array}$ & $\begin{array}{l}\text { Antibiotic + antimycotic } \\
\text { (unspecified) }\end{array}$ & \\
\hline
\end{tabular}

${ }^{\circ} \mathrm{C}$ degrees celsius, $L t d$. limited liability company, $\mathrm{mL}$ milliliter, $\mathrm{NaCl}$ sodium chloride, $P B S$ phosphate-buffered saline

${ }^{a}$ Pulmonary homograft decellularized according to the SynerGraft ${ }^{\mathrm{TM}}$ treatment protocol (CryoLife Inc., Kennesaw, Georgia, US) [54]

${ }^{b}$ Decellularization of pulmonary homograft performed at corlife oHG (corlife oHG, Hannover Medical School, Hannover, DE)

'Pulmonary homograft decellularized according to the patented process developed by AutoTissue Ltd. (AutoTissue GmbH, Berlin, DE) [27]

${ }^{\mathrm{d}}$ Pulmonary homograft decellularized according to the patented process developed by Tissue Regenix Ltd. (Tissue Regenix Ltd., Leeds, Yorkshire, UK) [28]

ePenicillin-streptomycin solution manufactured by Cedarlane Laboratories (PenStrep, Cedarlane Laboratories, Burlington, Ontario, CA)

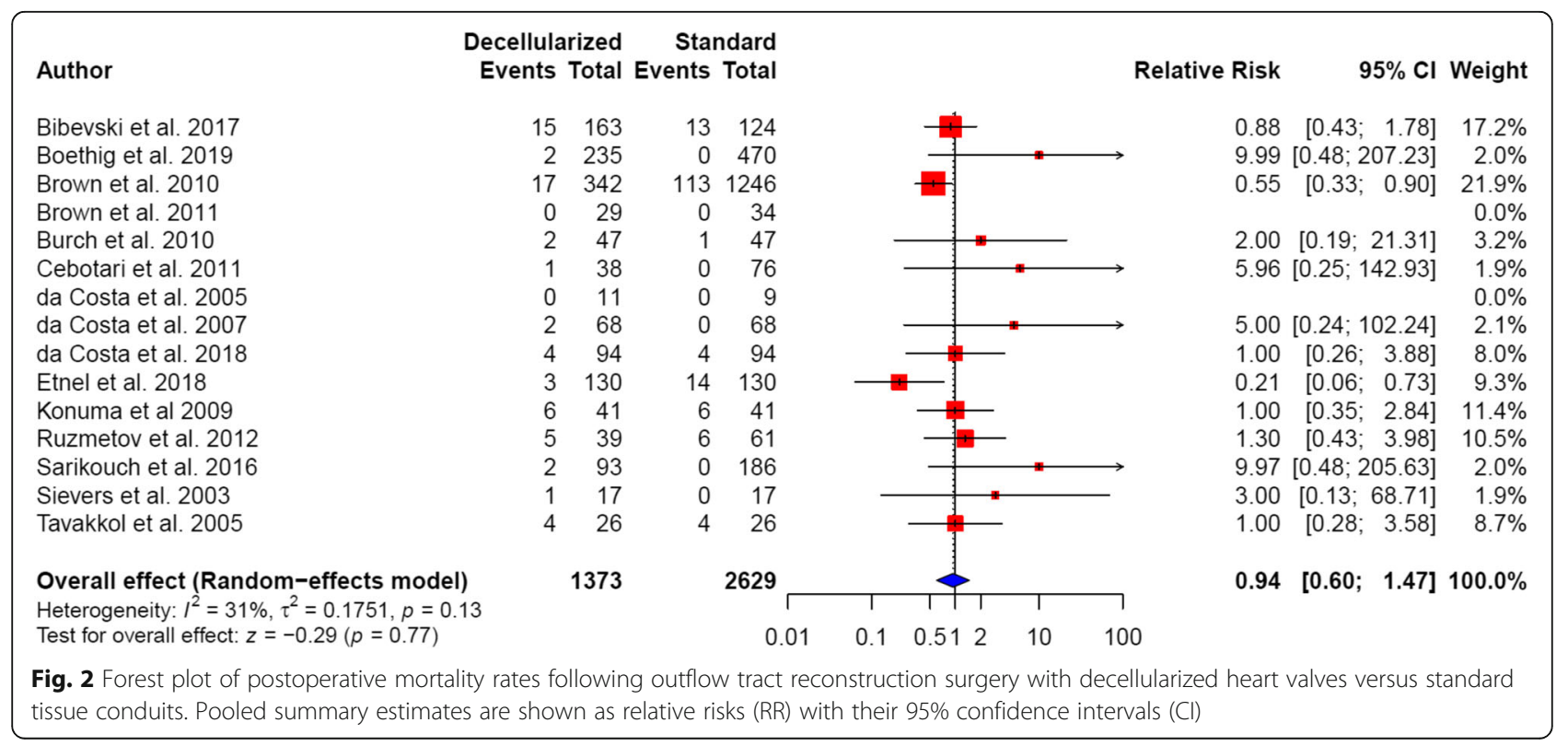




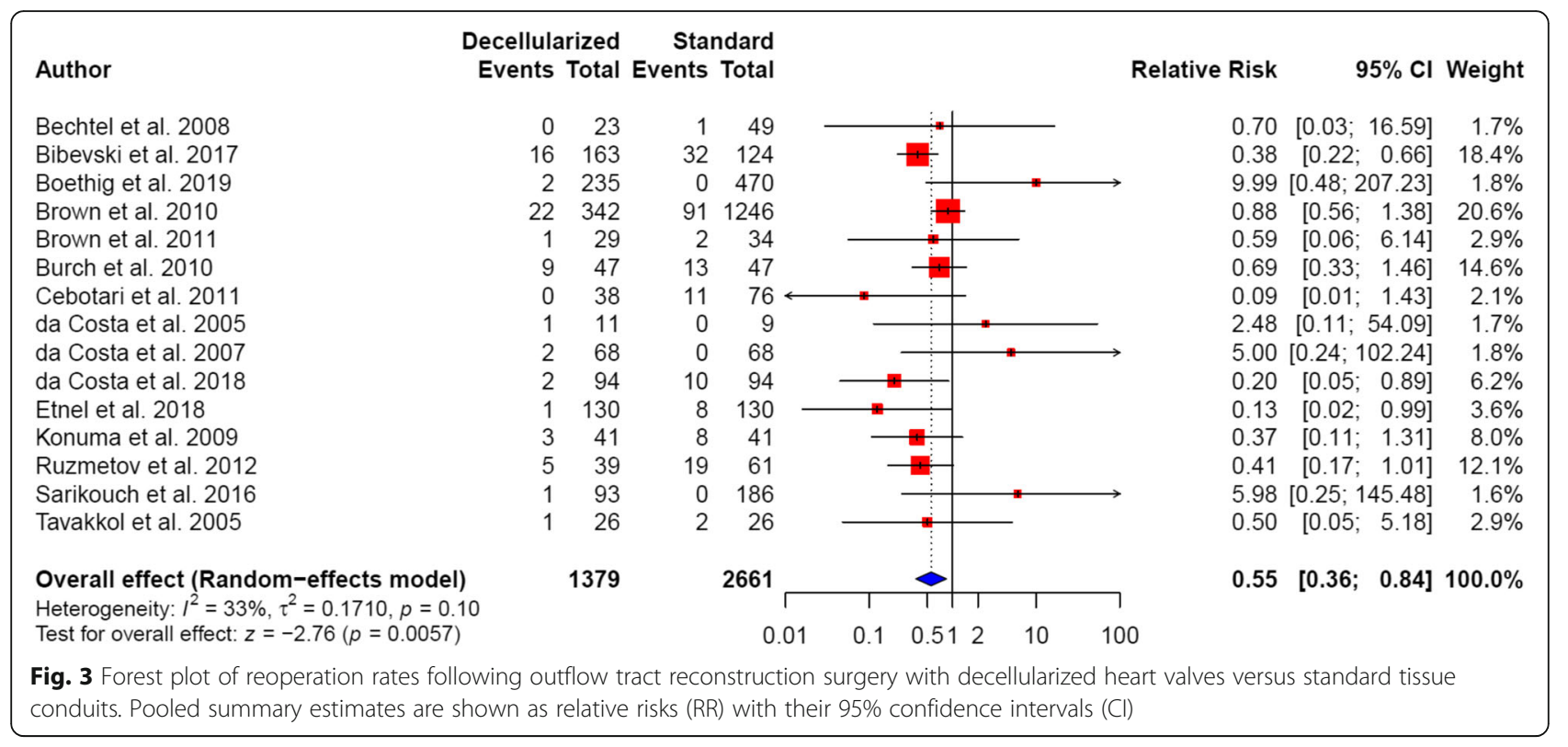

\section{Publication bias}

Funnel plot asymmetry was used to assess publication bias for postoperative mortality rates and reoperation rates within the analyzed studies (Figs. 4 and 5 respectively). Egger's regression test demonstrated significant publication bias for postoperative mortality rates $(p=0.004)$, with no publication bias observed for reoperation rates $(p=0.86)$.

\section{Sensitivity analysis}

For the purposes of this sensitivity analysis, six studies were excluded from the original analysis because they were either at moderate or serious risk of bias ( $n=5$ studies) [33, 34, $40,46,47,52]$ or had a disproportionately large sample size ( $n=1$ study) [43]. Nine studies were included in the final sensitivity analysis of postoperative mortality rates and reoperation rates [41, 42, 44, 45, 48-51, 53]. In comparison

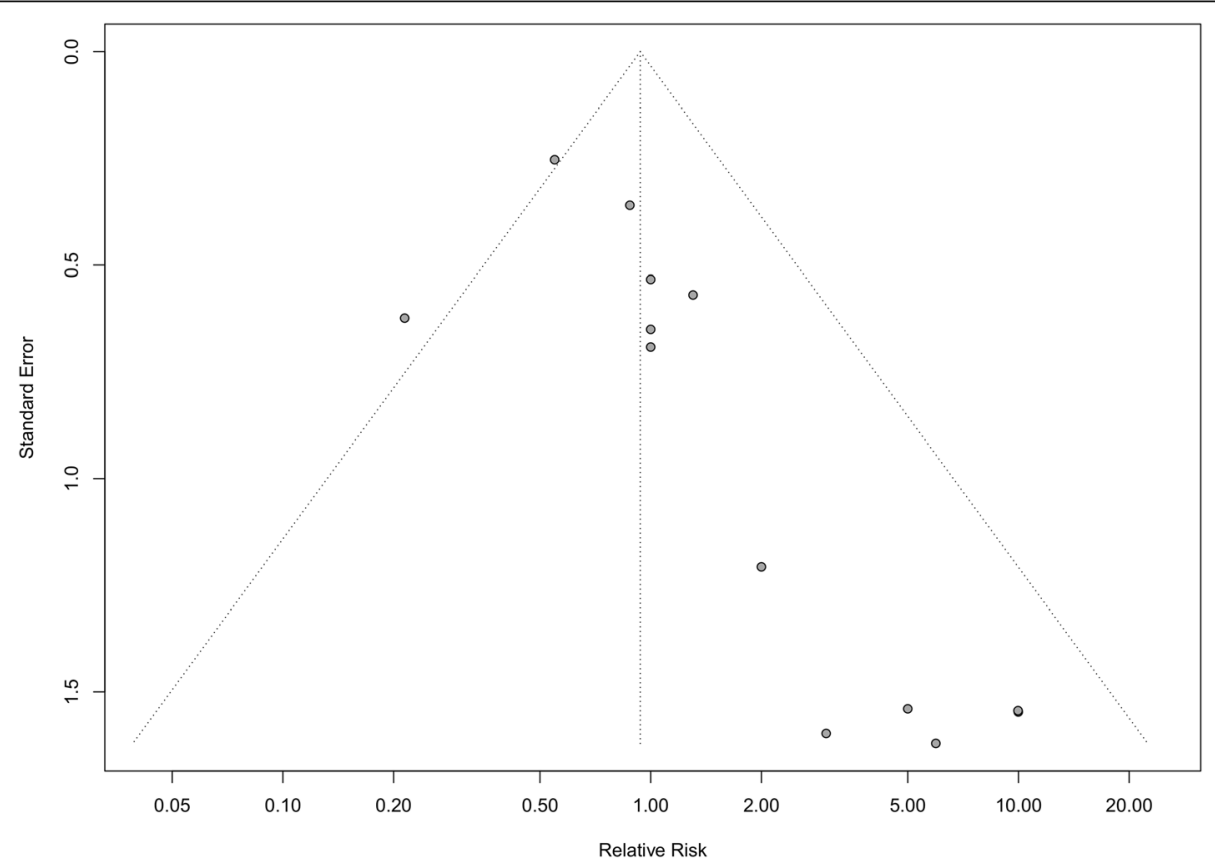

Fig. 4 Funnel plot for the assessment of publication bias of postoperative mortality with decellularized heart valves versus standard tissue conduits 


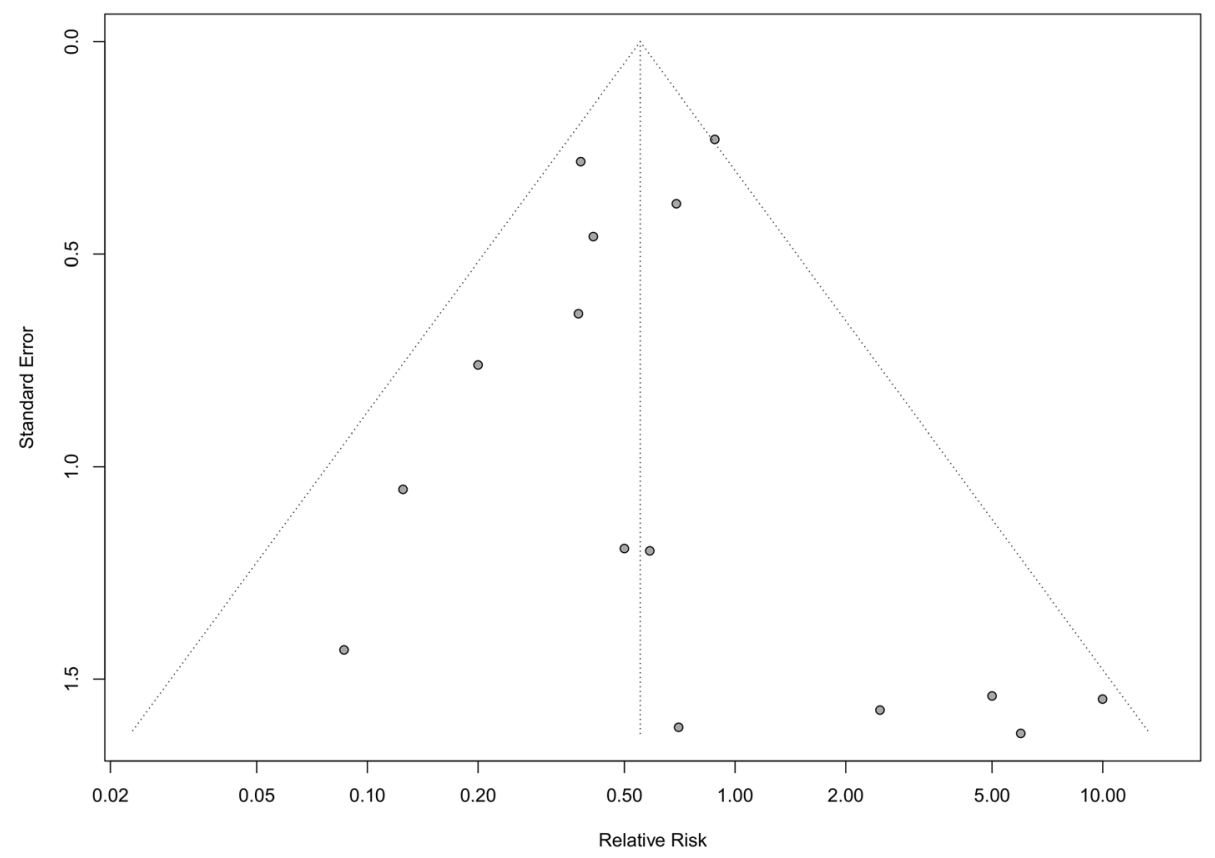

Fig. 5 Funnel plot for the assessment of publication bias of reoperation rates with decellularized heart valves versus standard tissue conduits

to standard tissue conduits, decellularized heart valves were associated with lower postoperative mortality (pooled RR 0.90, 95\% CI: 0.55 to $1.46 ; P=0.66$; Additional file 6) and significantly lower reoperation rates (pooled RR 0.44, 95\% CI: 0.30 to $0.63 ; P<0.0001$; Additional file 7$)$. There was no significant heterogeneity between the analyzed studies $\left(I^{2}=\right.$ $20 \%, P=0.27$ and $I^{2}=5 \%, P=0.39$ respectively).

\section{Secondary endpoints}

Transvalvular gradients were reported in all 17 articles. For patients with decellularized heart valves, 13 articles described either a reduction in the peak transvalvular gradient or a higher freedom from raised transvalvular gradients. Six of these articles, including the largest study by Brown et al., reached statistical significance $(p<0.05)$ [41, $43,46,47,49,53]$. In the four remaining articles, there was either an increase in the peak transvalvular gradient or lower freedom from raised transvalvular gradients. Only Bechtel and colleagues demonstrated the finding to be statistically significant $(p=0.049)$ [40].

The remainder of the secondary endpoints were irregularly reported. These endpoints included: valvular insufficiency [41, 53], valvular stenosis [43, 48, 49], conduit explantation [34], dysfunction [41, 48, 49, 51], and failure rates [43, 51], valvular diameter [39], area [39, 52], cusp mobility and retraction [33, 46], histological valvular analysis [33, 39, 46, 47], and adverse events such as infective endocarditis [34, 42, 43, 48] and valve thrombosis [43].

\section{Discussion}

This systematic review and meta-analysis included 17 studies, which consisted mostly of small studies, with few at low risk of bias. We found that patients with decellularized heart valves implanted within the right ventricular outflow tract had significantly lower reoperation rates and transvalvular gradients, when compared to patients with standard tissue conduits. In addition, the meta-analysis also demonstrated lower postoperative mortality rates among patients with decellularized heart valves, although this result was not statistically significant.

Tissue-engineered heart valves have the potential to revolutionize valve replacement surgery should they be able to overcome the limitations faced by prostheses in current clinical use [4-12]. Xenografts, unlike homografts, would serve as the ideal donor tissue type because of their virtually limitless availability [12]. It is therefore unfortunate that no studies of xenografts were included in this review. Nonetheless, during the screening of fulltext articles, 40 studies conducted using decellularized xenograft heart valves were identified but were excluded from the subsequent analysis because they were either not conducted in human subjects ( $n=29$ studies) or did not have a comparator group ( $n=11$ studies) and thus did not meet our inclusion criteria of human trials with a comparison group.

The aortic valve tends to require surgical intervention more frequently than the pulmonary valve because of higher rates of valvular dysfunction [55]. Therefore, the optimal tissue-engineered heart valve must be capable of 
withstanding the greater pressure demands of the left heart. None of the 17 studies included in this review described the experiences of decellularized valves in the left ventricular outflow tract position. However, during the screening of full-text articles, 15 studies of left ventricular outflow tract reconstructions were identified, although these texts were excluded from the subsequent analysis because their performance was either evaluated in non-human subjects ( $n=10$ studies) or they lacked a comparator group ( $n=5$ studies).

Despite the potential benefits of achieving a decellularized conduit for aortic valve replacement, many international groups have instead elected to focus on designing a graft for the pulmonary valve position because the native pulmonary valve may be transferred to the aortic position during the Ross procedure [27]. Although the Ross procedure has been life-changing for many, especially younger, patients, it remains a more complex operation than an aortic valve replacement [56]. Thus, the design of a prosthetic graft that is specifically intended for the left heart remains a priority.

In addition, a recent concept being explored in heart valve tissue-engineering is construct recellularization using autologous cells [32]. In essence, a decellularized construct is repopulated with the recipient's cells and then conditioned within a bioreactor ahead of surgical implantation [23, 24]. None of the studies assessed in this article combined a recellularization component with decellularization in their tissue-engineering technology. Much work is being undertaken to explore the capabilities of tissue-engineered scaffolds that are produced from decellularized xenografts and then recellularized using the future host's autologous cells [23, 24, 32]. Such a graft is expected to be living and thus have the potential to grow, repair, and remodel in situ [15-19].

There are several limitations with this review. Firstly, seven of the included studies were of low quality, with missing data or a lack of confounding being the predominant causes. Secondly, it is significant that only one study of xenograft performance within the left ventricular outflow tract position was identified during the systematic search [57]. Perhaps it is the catastrophic experiences of early xenograft studies that have resulted in the few, low-quality investigations of xenografts in human subjects [25, 58-61]. However, with further development in tissue-engineering technology, the in vitro performance of xenografts could excel to the point where they are brought to clinical trial in large, highquality studies. It is hypothesized that reducing the immunogenicity of the donor construct is central to achieving this goal. Osmotic shock and the use of enzymes that denature genetic material are useful for this process $[15,16,20]$. Lastly, it is noteworthy that only seven studies incorporated detergents in their decellularization protocol [33, 34, 42, 46-49], because detergent-use has also proven highly effective when incorporated in decellularization protocols $[13,14,17]$.

The number of permutations that affects the successfulness of a decellularization process is extensive. For example, small changes in variables such as the detergent combination or concentration, and the duration and temperature of incubation can have a profound impact on the effectiveness of a given protocol. Therefore, tissue-engineering technology should not be dismissed while ongoing laboratory work is being undertaken to refine and further develop decellularization protocols.

\section{Conclusions}

The published experiences of decellularized heart valves implanted within the right ventricular outflow tract of human subjects are typically small studies, in which the available data demonstrate significantly lower reoperation rates and transvalvular gradients, when compared to standard tissue conduits. No studies of tissueengineered xenografts or conduits implanted within the left ventricular outflow tract were able to be included in this review. Only seven studies incorporated detergents in their decellularization protocol and no studies examined a recellularization component. Thus, the exhaustive clinical potential of tissue-engineered heart valves remains to be elucidated. Future studies should rather aim to investigate the clinical utility of left ventricular outflow tract reconstructions using decellularized heart valves implanted within human subjects. In future, it is also recommended that studies within the heart valve tissue-engineering field consistently report standardized outcomes such as 30-day and one-year postoperative: mortality rates, reoperation rates, transvalvular gradients, valvular dysfunction, diameter and area, in addition to being of higher quality in terms of their design. The adoption of a standardized set of outcomes would allow for more accurate conclusions, particularly regarding patient safety, to be drawn about the undoubtable progress in development of decellularized heart valves and their resultant clinical performance.

\section{Supplementary information}

Supplementary information accompanies this paper at https://doi.org/10. 1186/s13019-020-01292-y.

Additional file 1. Criteria for awarding stars using the Newcastle-Ottawa Quality Assessment Scale [36].

Additional file 2. Methodological quality assessment for included fulltext observational cohort studies [36].

Additional file 3. Risk of bias assessment for included full-text nonrandomized interventional studies [37].

Additional file 4. Indications for outflow tract reconstructions in eligible full-text articles. 
Additional file 5. Primary endpoints (postoperative mortality and reoperations) in eligible full-text articles.

Additional file $\mathbf{6}$. Forest plot of postoperative mortality rates following outflow tract reconstruction surgery with decellularized heart valves versus standard tissue conduits. Studies at moderate or serious risk of bias and with a disproportionately large sample size have been excluded for the purposes of this sensitivity analysis. Pooled summary estimates are shown as relative risks (RR) with their $95 \%$ confidence intervals $(\mathrm{Cl})$.

Additional file 7. Forest plot of reoperation rates following outflow tract reconstruction surgery with decellularized heart valves versus standard tissue conduits. Studies at moderate or serious risk of bias and with a disproportionately large sample size have been excluded for the purposes of this sensitivity analysis. Pooled summary estimates are shown as relative risks (RR) with their 95\% confidence intervals (Cl).

\section{Abbreviations}

Cl: Confidence Interval; MeSH: Medical Subject Headings; P-value: Probability value; PRISMA: Preferred Reporting Items for Systematic Reviews and MetaAnalyses; RCT: Randomized Controlled Trial; ROBINS-I: Risk of Bias In NonRandomized Studies of Interventions; RR: Relative Risk

\section{Acknowledgements}

Not applicable.

\section{Authors' contributions}

SW conceived the idea for the systematic review and meta-analysis; SW and SB literature searched, systematically reviewed, quality assessed, extracted and analyzed the data; SW drafted the manuscript; SB, MG, PM and JC substantively revised the manuscript; All authors approved the submitted version and have agreed both to be personally accountable for the author's own contributions and to ensure that questions related to the accuracy or integrity of any part of the work, even ones in which the author was not personally involved, are appropriately investigated, resolved, and the resolution documented in the literature.

\section{Funding}

This work was supported by funding gratefully received from the Green Lane Research and Education Fund (Greenlane Research and Education Fund, Grafton, Auckland, NZ), and the National Heart Foundation of New Zealand (National Heart Foundation of New Zealand, Ellerslie, Auckland, NZ). SW received a postgraduate scholarship from the National Heart Foundation of New Zealand.

\section{Availability of data and materials}

The datasets used and analyzed during the current study are available from the corresponding author on reasonable request.

\section{Ethics approval and consent to participate}

Not applicable.

\section{Consent for publication}

Not applicable.

\section{Competing interests}

The authors declare that they have no competing interests.

\section{Author details}

${ }^{1}$ Department of Medicine, Faculty of Medical and Health Sciences, University of Auckland, Grafton, Auckland 1023, New Zealand. ${ }^{2}$ Adult Emergency Department, Auckland City Hospital, Auckland District Health Board, Grafton, Auckland 1023, New Zealand. ${ }^{3}$ Department of Surgery, Faculty of Medical and Health Sciences, University of Auckland, Grafton, Auckland 1023, New Zealand. ${ }^{4}$ Department of Medicine, Dunedin School of Medicine, University of Otago, Dunedin 9054, New Zealand. ${ }^{5}$ Green Lane Cardiothoracic Surgical Unit, Auckland City Hospital, Auckland District Health Board, Grafton, Auckland 1023, New Zealand.
Received: 26 May 2020 Accepted: 3 September 2020

Published online: 18 September 2020

\section{References}

1. Freling HG, Van Slooten YJ, Van Melle JP, Ebels T, Hoendermis ES, Berger RMF, et al. Pulmonary valve replacement: twenty-six years of experience with mechanical valvar prostheses. Ann Thorac Surg. 2015;99:905-10.

2. Pereira JJ, Lauer MS, Bashir M, Afridi I, Blackstone EH, Stewart WJ, et al. Survival after aortic valve replacement for severe aortic stenosis with low transvalvular gradients and severe left ventricular dysfunction. J Am Coll Cardiol. 2002;39(8):1356-63.

3. Schwarz F, Baumann P, Manthey J, Hoffmann M, Schuler G, Mehmel HC, et al. The effect of aortic valve replacement on survival. Circulation. 1982; 66(5):1105-10

4. Shin HJ, Kim YH, Ko JK, Park IS, Seo DM. Outcomes of mechanical valves in the pulmonic position in patients with congenital heart disease over a 20year period. Ann Thorac Surg. 2013;95:1367-71.

5. Stulak JM, Dearani JA, Burkhart HM, Connolly HM, Warnes CA, Suri RM, et al. The increasing use of mechanical pulmonary valve replacement over a 40year period. Ann Thorac Surg. 2010;90:2009-14.

6. Akhtar RP, Abid AR, Zafar H, Khan JS. Aniticoagulation in patients following prosthetic heart valve replacement. Ann Thorac Cardiovasc Surg. 2009;15:10-7.

7. Hering D, Piper C, Bergemann R, Hillenbach C, Dahm M, Huth C, et al. Thromboembolic and bleeding complications following St. Jude medical valve replacement: results of the German experience with low-intensity anticoagulation study. Chest. 2005:127:53-9.

8. Hirji SA, Kolkailah AA, Ramirez-Del Val F, Lee J, McGurk S, Pelletier M, et al. Mechanical versus bioprosthetic aortic valve replacement in patients aged 50 years and younger. Ann Thorac Surg. 2018;106(4):P1113-20.

9. Butany J, Soor GS, Chakrabarti M, Vukin I, Leong SW. Prosthetic heart valves, part I: identification and potential complications. Geriatr Aging. 2006;10:691-6.

10. Siddiqui RF, Abraham JR, Butany J. Bioprosthetic heart valves: modes of failure. Histopathology. 2009:55:135-44.

11. Dittrich S, Alexi-Meskishvili W, Yankah AC, Dähnert I, Meyer R, Hetzer R, et al. Comparison of porcine xenografts and homografts for pulmonary valve replacement in children. Ann Thorac Surg. 2000;70(3):717-22.

12. Angell WW, DeLanerolle P, Shumway NE. Valve replacement: present status of homograft valves. Prog Cardiovasc Dis. 1973;15(6):589-622.

13. Rieder E, Nigisch A, Dekan B, Kasimir MT, Mühlbacher F, Wolner E, et al. Granulocyte-based immune response against decellularized or glutaraldehyde cross-linked vascular tissue. Biomaterials. 2006;27(33):5634-42.

14. Rieder E, Seebacher G, Kasimir MT, Eichmair E, Winter B, Dekan B, et al. Tissue engineering of heart valves: Decellularized porcine and human valve scaffolds differ importantly in residual potential to attract monocytic cells. Circulation. 2005;111:2792-7.

15. Schenke-Layland K, Riemann I, Opitz F, König K, Halbhuber KJ, Stock UA Comparative study of cellular and extracellular matrix composition of native and tissue engineered heart valves. Matrix Biol. 2004;23(2):113-25.

16. Steinhoff $G$, Stock U, Karim N, Mertsching H, Timke A, Meliss RR, et al. Tissue engineering of pulmonary heart valves on allogenic acellular matrix conduits: in vivo restoration of valve tissue. Circulation. 2000;102(19 Suppl 3):III50-5.

17. Rieder E, Kasimir MT, Silberhumer G, Seebacher G, Wolner E, Simon P, et al. Decellularization protocols of porcine heart valves differ importantly in efficiency of cell removal and susceptibility of the matrix to recellularization with human vascular cells. J Thorac Cardiovasc Surg. 2004;127(2):399-405.

18. Tudorache I, Calistru A, Baraki H, Meyer T, Höffler K, Sarikouch S, et al. Orthotopic replacement of aortic heart valves with tissue-engineered grafts. Tissue Eng Part A. 2013;19(15-16):1686-94.

19. Dohmen PM. Clinical results of implanted tissue engineered heart valves. HSR Proc Intensive Care Cardiovasc Anesth. 2012:4(4):225-31.

20. Schenke-Layland K, Vasilevski O, Opitz F, König K, Riemann I, Halbhuber KJ, et al. Impact of decellularization of xenogeneic tissue on extracellular matrix integrity for tissue engineering of heart valves. J Struct Biol. 2003;143(3):201-8.

21. Dohmen PM, Lembcke A, Holinski S, Kivelitz D, Braun JP, Pruss A, et al. Midterm clinical results using a tissue-engineered pulmonary valve to reconstruct the right ventricular outflow tract during the Ross procedure. Ann Thorac Surg. 2007:84(3):729-36.

22. Iwai S, Torikai K, Coppin CM, Sawa Y. Minimally immunogenic decellularized porcine valve provides in situ recellularization as a stentless bioprosthetic valve. J Artif Organs. 2007;10(1):29-35. 
23. Converse GL, Buse EE, Neill KR, McFall CR, Lewis HN, VeDepo MC, et al. Design and efficacy of a single-use bioreactor for heart valve tissue engineering. J Biomed Mater Res - Part B Appl Biomater. 2017;105(2):249-59.

24. Tefft BJ, Choe JA, Young MD, Hennessy RS, Morse DW, Bouchard JA, et al. Cardiac valve bioreactor for physiological conditioning and hydrodynamic performance assessment. Cardiovasc Eng Technol. 2019;10:80-94.

25. Simon P, Kasimir MT, Seebacher G, Weigel G, Ullrich R, Salzer-Muhar U, et al. Early failure of the tissue engineered porcine heart valve SYNERGRAFT ${ }^{\mathrm{M}}$ in pediatric patients. Eur J Cardio-thoracic Surg. 2003;23(6):1002-6.

26. Konertz W, Dohmen PM, Liu J, Beholz S, Dushe S, Posner S, et al. Hemodynamic characteristics of the matrix $P$ decellularized xenograft for pulmonary valve replacement during the Ross operation. J Heart Valve Dis. 2005;14(1):78-81.

27. Konertz W, Dohmen P. Method for decellularising foreign material for the production of bioprostheses 2004;1(19):12-17. Available from: https:/patentimages. storage.googleapis.com/63/50/48/aac2bdc35e50ce/CA2428880C.pdf.

28. Bolland F, Southgate J, Korossis S, Ingham E. Decellularisation of tissue matrices for bladder implantation. 2015;2(12). Available from: https:/patentimages. storage.googleapis.com/59/4a/27/acc29a59d8b0c5/US9180144.pdf.

29. Dohmen PM, Da Costa F, Holinski S, Lopes SV, Yoshi S, Reichert LH, et al. Is there a possibility for a glutaraldehyde-free porcine heart valve to grow? Eur Surg Res. 2006;38:54-61.

30. Helder MRK, Stoyles NJ, Tefft BJ, Hennessy RS, Hennessy RRC, Dyer R, et al. Xenoantigenicity of porcine decellularized valves. J Cardiothorac Surg. 2017; 12(1):56.

31. Park CS, Kim YJ, Lee JR, Lim HG, Chang JE, Jeong S, et al. Anticalcification effect of a combination of decellularization, organic solvents and amino acid detoxification on glutaraldehyde-fixed xenopericardial heart valves in a large-animal long-term circulatory model. Interact Cardiovasc Thorac Surg. 2017:25(3):391-9.

32. Cebotari S, Lichtenberg A, Tudorache I, Hilfiker A, Mertsching H, Leyh R, et al. Clinical application of tissue engineered human heart valves using autologous progenitor cells. Circulation. 2006;114(1 Suppl):1132-7.

33. Costa F, Dohmen P, Vieira E, Pereira EWL, Matsuda CN, Lopes SV, et al. Ross operation with decelularized pulmonary allografts: medium-term results. Brazilian J Cardiovasc Surg. 2007:22(4):454-62.

34. Sarikouch S, Horke A, Tudorache I, Beerbaum P, Westhoff-Bleck M, Boethig $D$, et al. Decellularized fresh homografts for pulmonary valve replacement: a decade of clinical experience. Eur J Cardio-thoracic Surg. 2016;50(2):281-90.

35. Moher D, Liberati A, Tetzlaff J, Altman DG, Altman D, Antes G, et al. Preferred reporting items for systematic reviews and meta-analyses: the PRIS MA statement. PLoS Med. 2009:6(7):e1000097.

36. Wells G, Shea B, O'Connell D, Peterson J. The Newcastle-Ottawa scale (NOS) for assessing the quality of nonrandomised studies in meta-analyses. Ottawa: Ottawa Hospital Research Institute. 2000 [cited 2020 Apr 23]. Available from: http://www.ohri.ca/programs/clinical_epidemiology/nosgen.pdf.

37. Sterne JA, Hernán MA, Reeves BC, Savović J, Berkman ND, Viswanathan M, et al. ROBINS-I: a tool for assessing risk of bias in non-randomised studies of interventions. BMJ. 2016:355:1-7.

38. Higgins JPT, Thompson SG, Deeks JJ, Altman DG. Measuring inconsistency in meta-analyses. Br Med J. 2003;327:557-60

39. Bechtel JFM, Erasmi AW, Petersen M, Stierle U, Sievers H-H, Gellissen J, et al. Mid-term findings on echocardiography and computed tomography after RVOT-reconstruction: comparison of decellularized (SynerGraft) and conventional allografts. Eur J Cardio-thoracic Surg. 2005;27(3):410-5.

40. Bechtel M, Stierle U, Sievers H-H. Fifty-two months' mean follow up of decellularized SynerGraft ${ }^{\text {TM }}$ - treated pulmonary valve allografts. J Heart Valve Dis. 2008;17(1):98-104

41. Bibevski S, Ruzmetov M, Fortuna RS, Turrentine MW, Brown JW, Ohye RG. Performance of SynerGraft Decellularized pulmonary allografts compared with standard cryopreserved allografts: results from multiinstitutional data. Ann Thorac Surg. 2017;103(3):869-74.

42. Boethig D, Horke A, Hazekamp M, Meyns B, Rega F, Van Puyvelde J, et al. A European study on decellularized homografts for pulmonary valve replacement: initial results from the prospective ESPOIR trial and ESPOIR registry datat. Eur J Cardio-Thoracic Surg. 2019;56(3):503-9.

43. Brown JW, Elkins RC, Clarke DR, Tweddell JS, Huddleston CB, Doty JR, et al. Performance of the CryoValve* SG human decellularized pulmonary valve in 342 patients relative to the conventional Cryovalve at a mean follow-up of four years. J Thorac Cardiovasc Surg. 2010;139(2):339-48 Available from: https://doi.org/10.1016/j.jtcvs.2009.04.065.
44. Brown JW, Ruzmetov M, Eltayeb O, Rodefeld MD, Turrentine MW. Performance of SynerGraft decellularized pulmonary homograft in patients undergoing a Ross procedure. Ann Thorac Surg. 2011;91(2):416-23.

45. Burch PT, Kaza AK, Lambert LM, Holubkov R, Shaddy RE, Hawkins JA. Clinical performance of decellularized cryopreserved valved allografts compared with standard allografts in the right ventricular outflow tract. Ann Thorac Surg. 2010;90(4):1301-6

46. Cebotari S, Tudorache I, Ciubotaru A, Boethig D, Sarikouch S, Goerler A, et al. Use of fresh decellularized allografts for pulmonary valve replacement may reduce the reoperation rate in children and young adults: Early report. Circulation. 2011;124(11 SUPPL. 1):115-23.

47. Affonso Da Costa FD, Dohmen PM, Duarte D, Von Glenn C, Lopes SV, Haggi Filho $\mathrm{H}$, et al. Immunological and echocardiographic evaluation of decellularized versus cryopreserved allografts during the Ross operation. Eur J Cardio-thoracic Surg. 2005;27(4):572-8.

48. da Costa FDA, Etnel JRG, Charitos El, Sievers HH, Stierle U, Fornazari D, et al. Decellularized versus standard pulmonary allografts in the Ross procedure: propensity-matched analysis. Ann Thorac Surg. 2018;105(4):1205-13. Available from: https://doi.org/10.1016/j.athoracsur.2017.09.057.

49. Etnel JRG, Suss PH, Schnorr GM, Veloso M, Colatusso DF, Filho EMB, et al. Fresh decellularized versus standard cryopreserved pulmonary allografts for right ventricular outflow tract reconstruction during the Ross procedure: a propensity-matched study. Eur J Cardio-Thoracic Surg. 2018;54(3):434-40.

50. Konuma T, Devaney EJ, Bove EL, Gelehrter S, Hirsch JC, Tavakkol Z, et al. Performance of CryoValve SG Decellularized pulmonary allografts compared with standard cryopreserved allografts. Ann Thorac Surg. 2009;88(3):849-55 Available from: https://doi.org/10.1016/j.athoracsur.2009.06.003.

51. Ruzmetov M, Shah JJ, Geiss DM, Fortuna RS. Decellularized versus standard cryopreserved valve allografts for right ventricular outflow tract reconstruction: a single-institution comparison. J Thorac Cardiovasc Surg [Internet]. 2012;143(3):543-9 Available from: https://doi.org/10.1016/j.jtcvs. 2011.12.032.

52. Sievers HH, Stierl U, Schmidtke C, Bechtel M. Decellularized pulmonary homograft (SynerGraft) for reconstruction of the right ventricular outflow tract: first clinical experience. Z Kardiol. 2003;92(1):53-9.

53. Tavakkol Z, Gelehrter S, Goldberg CS, Bove EL, Devaney EJ, Ohye RG. Superior durability of synergraft pulmonary allografts compared with standard cryopreserved allografts. Ann Thorac Surg. 2005;80(5):1610-4

54. O'Brien MF, Goldstein S, Walsh S, Black KS, Elkins R, Clarke D. The SynerGraft valve: a new acellular (nonglutaraldehyde-fixed) tissue heart valve for autologous recellularization first experimental studies before clinical implantation. Semin Thorac Cardiovasc Surg. 1999;11(4 Suppl 1):194-200.

55. Antonini-Canterin F, Huang G, Cervesato E, Faggiano P, Pavan D, Piazza R, et al. Symptomatic aortic stenosis: does systemic hypertension play an additional role? Hypertension. 2003;41(6):1268-72.

56. Mazine A, David TE, Rao V, Hickey EJ, Christie S, Manlhiot C, et al. Long-term outcomes of the ross procedure versus mechanical aortic valve replacement. Circulation. 2016:134:576-85.

57. Goldstein S, Clarke DR, Walsh SP, Black KS, O'brien MF. Transpecies heart valve transplant: advanced studies of a bioengineered xeno-autograft. Ann Thorac Surg. 2000;70(6):1962-9.

58. Voges I, Bräsen JH, Entenmann A, Scheid M, Scheewe J, Fischer G, et al. Adverse results of a decellularized tissue-engineered pulmonary valve in humans assessed with magnetic resonance imaging. Eur J Cardio-thoracic Surg. 2013:44(4):e272-9.

59. Christ T, Paun AC, Grubitzsch H, Holinski S, Falk V, Dushe S. Long-term results after the Ross procedure with the decellularized AutoTissue matrix P® bioprosthesis used for pulmonary valve replacement. Eur J CardioThoracic Surg. 2019;55(5):885-92.

60. Perri G, Polito A, Esposito C, Albanese SB, Francalanci P, Pongiglione G, et al. Early and late failure of tissue-engineered pulmonary valve conduits used for right ventricular outflow tract reconstruction in patients with congenital heart disease. Eur J Cardio-Thoracic Surg. 2012;41:1320-5.

61. Rüffer A, Purbojo A, Cicha I, Glöckler M, Potapov S, Dittrich S, et al. Early failure of xenogenous de-cellularised pulmonary valve conduits - a word of caution! Eur J Cardio-Thoracic Surg. 2010;38:78-85.

\section{Publisher's Note}

Springer Nature remains neutral with regard to jurisdictional claims in published maps and institutional affiliations. 